\title{
Bilevel Programming, Equilibrium, and Combinatorial Problems with Applications to Engineering
}

\author{
Vyacheslav Kalashnikov, ${ }^{1,2,3}$ Timothy I. Matis, ${ }^{4}$ \\ José Fernando Camacho Vallejo, ${ }^{5}$ and Sergii V. Kavun ${ }^{6}$ \\ ${ }^{1}$ Department of Systems \& Industrial Engineering, Tecnológico de Monterrey (ITESM), Campus Monterrey, \\ Avenida Eugenio Garza Sada 2501 Sur, 64849 Monterrey, NL, Mexico \\ ${ }^{2}$ Department of Social Modeling, Central Economics \& Mathematics Institute (CEMI), Russian Academy of Sciences, \\ Nakhimovsky pr. 47, Moscow 117418, Russia \\ ${ }^{3}$ Department of Computing, Sumy State University, Rimsky-Korsakov St. 2, Sumy 40007, Ukraine \\ ${ }^{4}$ Department of Industrial Engineering, Texas Tech University, P.O. Box 43061, Lubbock, TX 79409, USA \\ ${ }^{5}$ Facultad de Ciencias Físico-Matemáticas, Universidad Autónoma de Nuevo León, Avenida Universidad S/N, \\ 66450 San Nicolás de los Garza, NL, Mexico \\ ${ }^{6}$ Kharkiv Institute of Banking of the University of Banking of the National Bank of Ukraine, \\ Peremogy Avenue 55, Kharkiv 61174, Ukraine \\ Correspondence should be addressed to Vyacheslav Kalashnikov; kalash@itesm.mx
}

Received 16 September 2014; Accepted 16 September 2014

Copyright (c) 2015 Vyacheslav Kalashnikov et al. This is an open access article distributed under the Creative Commons Attribution License, which permits unrestricted use, distribution, and reproduction in any medium, provided the original work is properly cited.

Although a wide range of applications fit the bilevel programming framework, real-life implementations are scarce, due mainly to the lack of efficient algorithms for tackling medium- and large-scale bilevel programming problems. Solving a bilevel (more generally, hierarchical) optimization problem, even in its simplest form, is a difficult task. A lot of different alternative methods may be used based on the structure of the problem analyzed, but there is no general method that guarantees convergence, performance, or optimality for every type of problem.

Many new ideas appeared and were discussed in works of plenty of authors. Among them, we would name Dempe [1], Dempe et al. [2], Dempe and Dutta [3], Dewez et al. [4], Thi et al. [5], and Vicente and Calamai [6], whose works have developed various ways of reducing original bilevel programming problems to equivalent single level ones, thus making their solution somewhat an easier task for conventional mathematical programming software packages.

Mixed-integer bilevel programming problems (with part of variables at the upper and/or lower level being integer/
Boolean ones) are even harder for the well-known conventional optimization techniques. For instance, a usual replacement of the lower level optimization problem with the corresponding Karush-Kuhn-Tucker (KKT) conditions may not work if some lower level variables are not continuous. Therefore, solid theoretical base is necessary to be found, in order to propose efficient algorithmic procedures aimed at finding local or global solutions of such a problem.

A great amount of new applied problems in the area of energy networks have recently arisen that can be efficiently solved only as mixed-integer bilevel programs. Among them are the natural gas cash-out problem, the deregulated electricity market equilibrium problem, biofuel problems, problem of designing coupled energy carrier networks, and so forth, if we mention only part of such applications. Bilevel models to describe migration processes are also in the running of the most popular new themes in the area of bilevel programming.

This special volume comprises papers dealing with three main themes: bilevel programming, equilibrium models, and combinatorial (integer programming) problems and their applications to engineering. 
The special issue opens with a survey paper "Bilevel programming and applications" by S. Dempe et al. that sums up some recent and new directions and results of the development of the mathematical methods aimed at the solution of bilevel programs of different types and their applications to real-life problems.

One of such applications is the well-known bilevel programming problem arising in the natural gas industry and related to the solution of the important imbalance cashout problem (a good part of the above-mentioned survey paper is devoted to this area). Since the stochastic statement of the problem in question needs the generation of scenario trees with a very large number of branches and arcs, the reduction of the quantity of common variables is an important challenge. This item is analyzed and solved by proposing new methods of grouping the variables (classifying and representing various groups of states with respect to the natural gas prices) in the article "US natural gas market classification using pooled regression" by V. V. Kalashnikov et al. The techniques proposed in this paper help classify regions or states into groups or classes that share similar regression parameters. Once obtained, these groups may be used to make assumptions about corresponding natural gas prices in further studies.

One of the principal management problems dealt with in numerous organizations and institutions in the public sector is to decide how to invest and manage funds available to support potential research and development projects in various areas. A classification of the portfolio problem distinguishes two types: (a) static and (b) dynamic. In (a) only projects proposals for funding are involved, while in (b) at certain moments some active projects are withdrawn from the portfolio and some inactive projects are activated. Within this framework, a social project is a group of tasks or activities consuming funds, carried out during a given period of time in one or more regions, with an impact on the objectives set by an organization, and focused on providing solution to problems or needs of the society. The paper "Selecting large portfolios of social projects in public organizations" by I. Litvinchev et al. models the task as a two-objective mixedinteger linear programming problem. The model supports both complete (all or nothing) and partial (a certain amount from a given interval of funding) resource allocation policies. Numerical results for large scale problem instances are also presented. The computational experiment demonstrates that increasing the number of projects subject to funds reduction results in a decrease in both objectives. However, if the number of projects subject to funds reduction is fixed but the strength of funds reduction is increased, one of the objectives may grow at the expense of a larger drop in another.

A capacitated fixed-cost facility location problem with transportation choices (CFCLP-TC) is studied in the paper "Variations in the flow approach to CFCLP-TC for multiobjective supply chain design" by M. P. Hertwin et al. The problem based on a production network of two echelons with multiple plants, a set of potential distribution centers and customers, is formulated as an optimization model with two objective functions with time and cost as variables. The main contribution of this paper is in an extension of the existing approaches to the supply of products to customers through multiple sources, the direct flow between plants and customers, and the product flow between distribution centers. Based upon these approaches, the authors generate mathematical programming models and propose solution methods by the $\varepsilon$-constraint approach. Namely, they generate Pareto boundaries and thus compare each of those approaches with the original model. The models are implemented in GAMS and solved with CPLEX.

Another work presenting a novel capacitated model for supply chain network design is the paper "Design and optimization of capacitated supply chain networks including quality measures" by K. K. Castillo-Villar et al. The model in question (abbreviated as SCND-COQ) is described as a mixed-integer nonlinear programming problem, which can be used at a strategic planning level to design a supply chain network that maximizes the total profit subject to meeting an overall quality level of the final product at the minimum costs. To process the problem, the authors propose five combinatorial optimization algorithms based on nonlinear optimization, heuristic, and metaheuristic approaches, which are used to solve realistic instances of practical size. The computational results demonstrate that the state and individual representation in the simulated annealing and the genetic algorithm, respectively, has a significant impact on the improvement of the solution quality.

A problem of packing a limited number of unequal circles in a fixed size rectangular container is considered in the paper "Integer programming formulations for approximate packing circles in a rectangular container" by I. Litvinchev and E. L. O. Espinosa. The aim of this research is to maximize the (weighted) number of circles placed into the container or minimize the waste. This problem has numerous applications in logistics, including production and packing for the textile, apparel, naval, automobile, aerospace, and food industries. Frequently the problem is formulated as a nonconvex continuous optimization problem, which is solved by heuristic techniques combined with local search procedures. The authors propose new formulations for an approximate solution of the packing problem. The container is approximated by a regular grid and the nodes of the grid are considered as potential positions for assigning centers of the circles. The packing problem is then stated as a large scale linear $0-1$ optimization problem. The binary variables represent the assignment of centers to the nodes of the grid. Nesting circles inside one another are also considered. The resulting binary problem is then solved by commercial software. Numerical results are presented to demonstrate the efficiency of the proposed approach and compared with known results.

The paper "A heuristic procedure for a ship routing and scheduling problem with variable speed and discretized time windows" by K. K. Castillo-Villar et al. develops a heuristic algorithm solving a routing and scheduling problem for tramp shipping with discretized time windows. The problem consists of determining the set of cargoes that should be served by each ship, as well as the arrival, departure, and waiting times at each port, while minimizing the total costs. The heuristic proposed is based on a variable neighborhood search, considering a number of neighborhood structures 
to find a solution to the problem. The authors present computational results, and, for comparison purposes, they consider instances that can be solved directly by CPLEX to test the performance of the proposed heuristic. The heuristic achieves good solution qualities within reasonable computational times. The obtained numerical results are encouraging and make the presented heuristic quite promising to be useful when solving large real-size examples.

It is well-known that modern approaches to the development of a national economy are often characterized with an imbalanced inflation of some economic branches leading to a disproportional socioeconomic territories development (SETD). Such disproportions, together with other similar factors, frequently result in a lack of economic integrity, various regional crises, and a low rate of the economic and territorial growth. Those disproportions may also conduce to an inadequate degree of the interregional collaboration. The paper "Simulation of territorial development based on fiscal policy tools" by R. Brumnik et al. proposes some new ways of regulating imbalances in the territorial development making use of the fiscal policy tools. The latter can immediately reduce the amplitude of economic cycle fluctuations and provide a stable development of the economic state system. The same approach is applied to control the processes of transformation of the tax legislation and tax relations, as well as the levying and redistribution of the recollected taxes among the territories' budgets (this approach is also known as a tax policy). To resume, this paper describes comprehensive models of financial regulation of the socioeconomic territorial development that can help in estimating and choosing the right financial policy parameters. The research highlights the following conclusions: analysis of the predictive dynamics of socioeconomic development territories, in the case of the implementation of an optimistic scenario of tax revenue, demonstrates the effectiveness of the adopted stabilization policy.

Though a bit off the special issue's general stream, still interesting results of research are provided by the article "The solution of fourth order boundary value problem arising out of the beam-column theory using Adomian decomposition method" by O. Kelesoglu. In this study, Adomian decomposition method (ADM) is applied to a linear nonhomogeneous boundary value problem arising from the beam-column theory. The obtained results of numerical experiments are presented in tables and illustrated with graphs. The proposed approach provides a rapidly converging method, with the approximate solutions tending to the exact solution of the problem in question. This fact characterizes the method as appropriate and reliable for such kind of problems.

We hope that readers of this special issue will find not only new ideas and algorithms dealing with the difficult applied problems like supply chain design, assignment problems, bilevel programming models, and so forth, but also some interesting results related to regression analysis, as well as the modern simulation techniques.

\section{Acknowledgments}

A special gratitude is also due to the long list of referees, whose valuable comments and suggestions have helped us a lot in improving our results and/or presentation style. The research activity of Vyacheslav Kalashnikov was financially supported by Tecnológico de Monterrey (ITESM), Campus Monterrey, and by the SEP-CONACYT Project CB-201301-221676, Mexico. The research activity of José Fernando Camacho Vallejo was financially supported by the Project PROMEP/103.5/10/3889, the Academic Groups Research Project PROMEP/103.5/12/4953, and the Autonomous University of Nuevo Leon (UANL) within the Support Program for Scientific Research and Technology (PAICYT) within the Project CE960-11.

Vyacheslav Kalashnikov
Timothy I. Matis
José Fernando Camacho Vallejo
Sergii V. Kavun

\section{References}

[1] S. Dempe, Foundations of Bilevel Programming, Springer, Heidelberg, Germany, 2002.

[2] S. Dempe, B. S. Mordukhovich, and A. B. Zemkoho, "Necessary optimality conditions in pessimistic bilevel programming," Optimization, vol. 63, no. 4, pp. 505-533, 2014.

[3] S. Dempe and J. Dutta, "Is bilevel programming a special case of a mathematical program with complementarity constraints?" Mathematical Programming A, vol. 131, pp. 37-48, 2012.

[4] S. Dewez, M. Labbé, P. Marcotte, and G. Savard, "New formulations and valid inequalities for a bilevel pricing problem," Operations Research Letters, vol. 36, no. 2, pp. 141-149, 2008.

[5] L. Thi, T. Duc, and P. Dinh, "A DC programming approach for a class of bilevel programming problems and its application in portfolio selection," Numerical Algebra, Control and Optimization, vol. 2, no. 1, pp. 167-185, 2012.

[6] L. N. Vicente and P. H. Calamai, "Bilevel and multilevel programming: a bibliography review," Journal of Global Optimization, vol. 5, no. 3, pp. 291-306, 1994. 


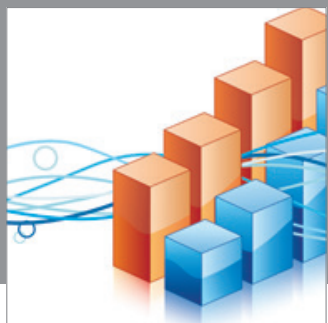

Advances in

Operations Research

mansans

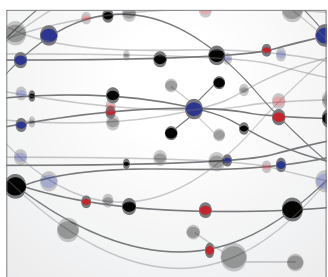

The Scientific World Journal
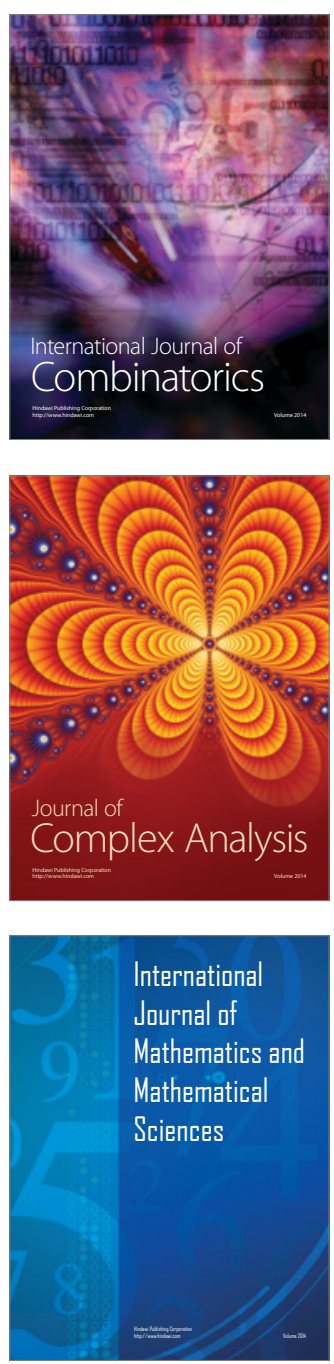
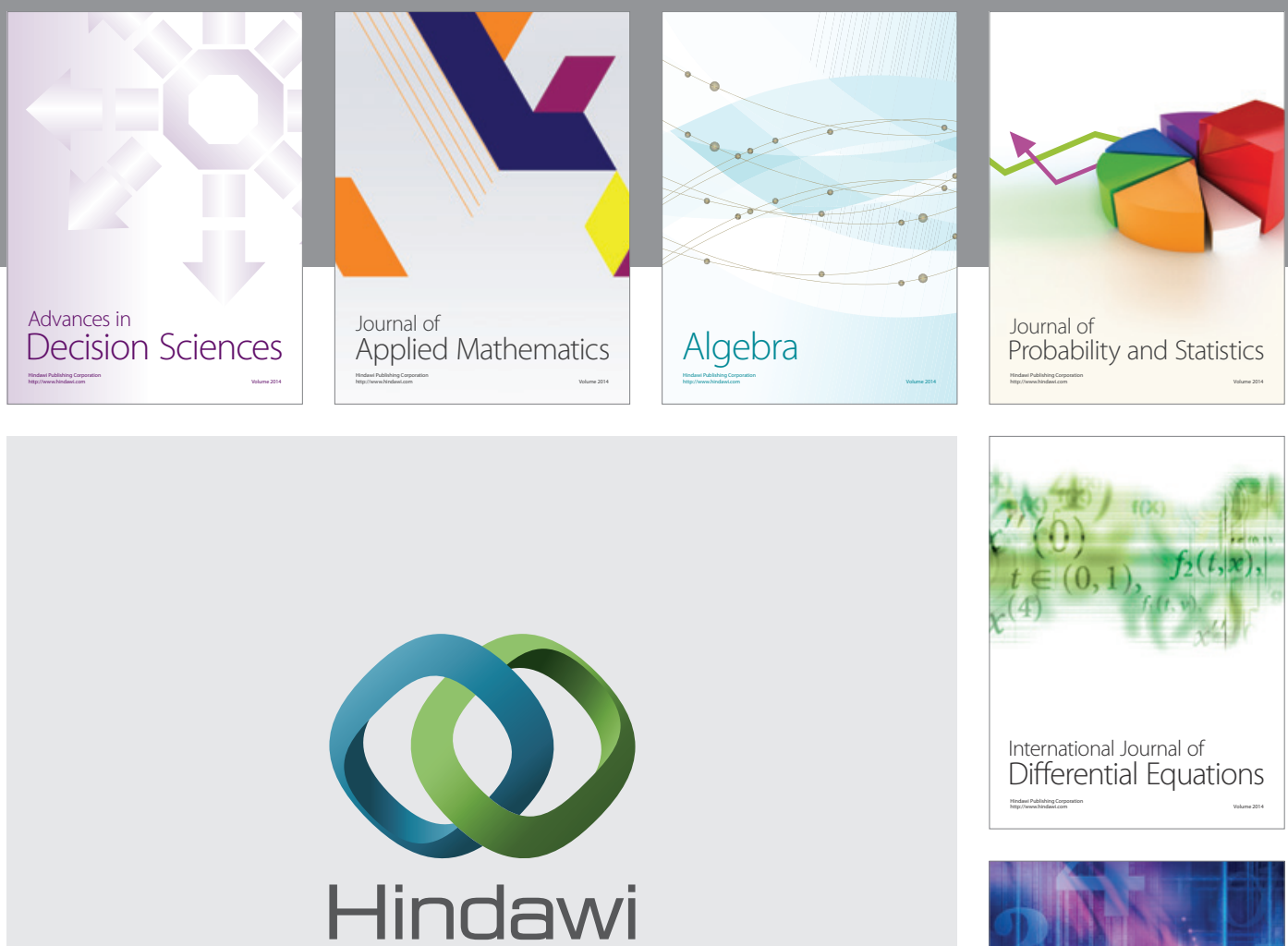

Submit your manuscripts at http://www.hindawi.com
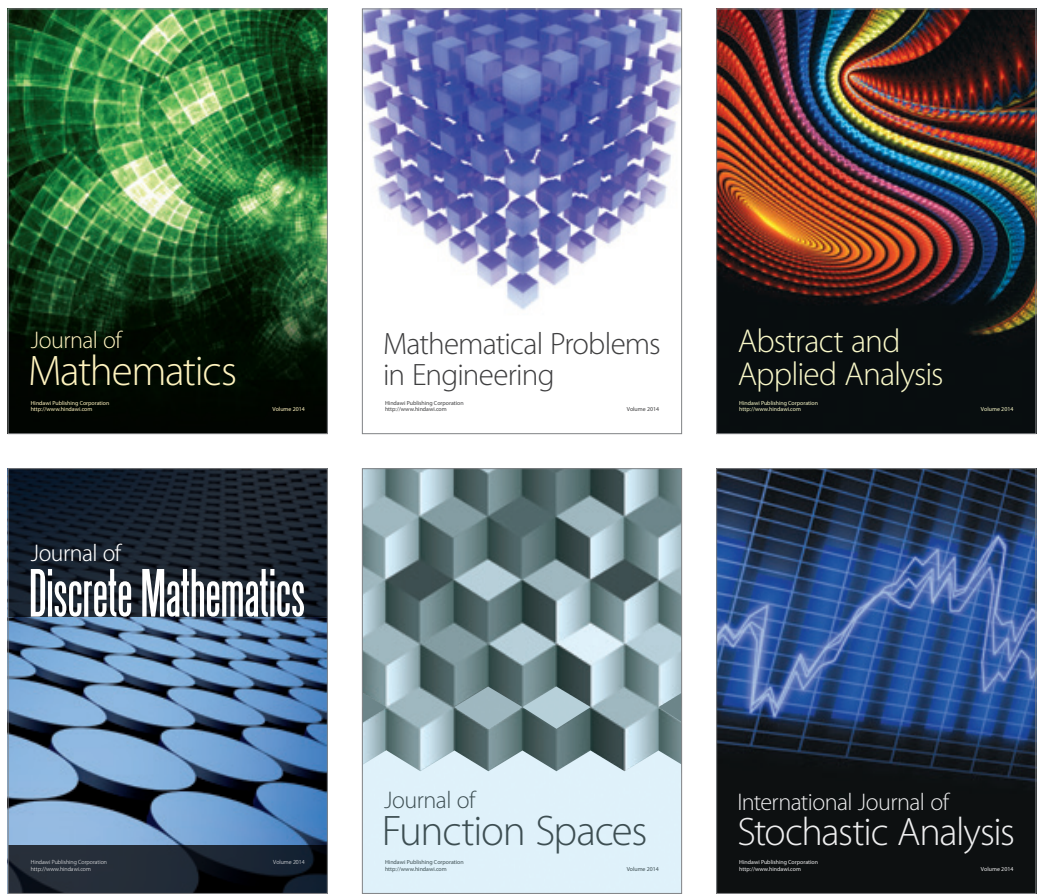

Journal of

Function Spaces

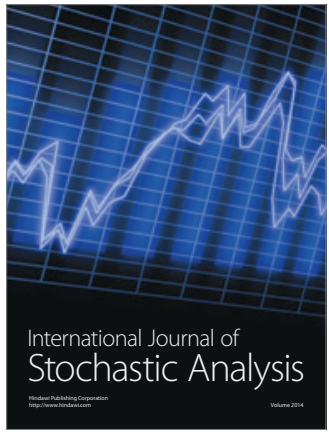

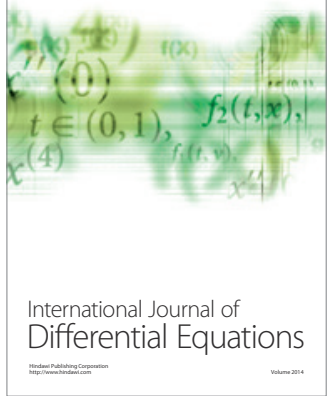
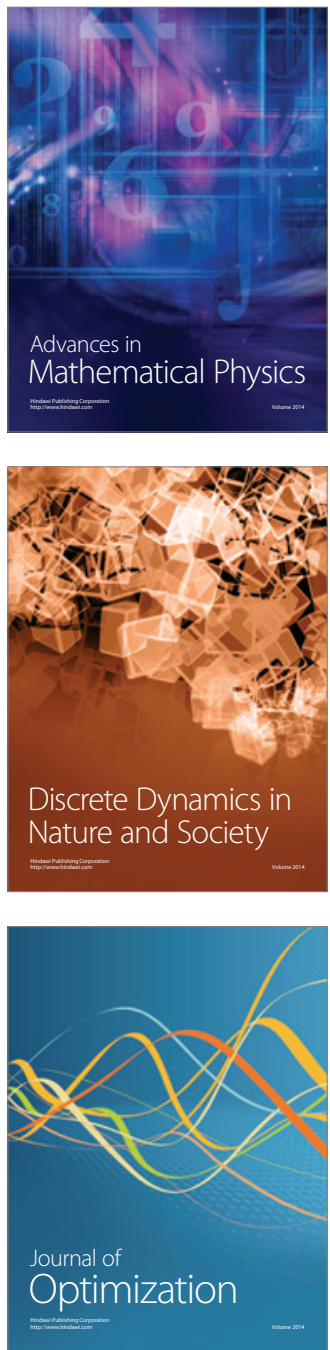